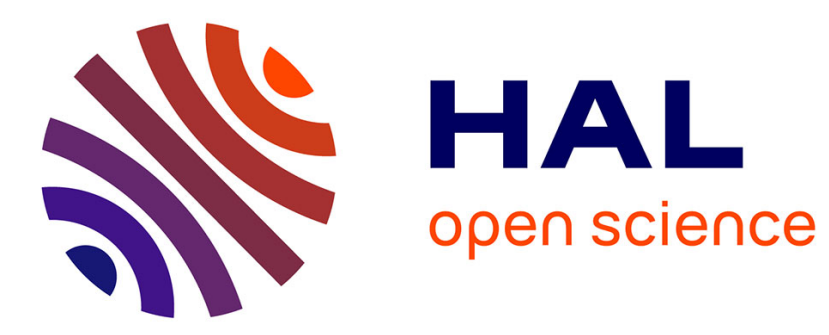

\title{
Le Parlement européen: tensions entre efficacité institutionnelle et démocratie
}

Nathalie Brack, Olivier Costa

\section{To cite this version:}

Nathalie Brack, Olivier Costa. Le Parlement européen: tensions entre efficacité institutionnelle et démocratie. Hérodote - Revue de géographie et de géopolitique, 2017, Menaces sur l'Europe, 164, pp.199-199. 10.3917/her.164.0199 . halshs-01590349

\section{HAL Id: halshs-01590349 \\ https://shs.hal.science/halshs-01590349}

Submitted on 19 Sep 2017

HAL is a multi-disciplinary open access archive for the deposit and dissemination of scientific research documents, whether they are published or not. The documents may come from teaching and research institutions in France or abroad, or from public or private research centers.
L'archive ouverte pluridisciplinaire HAL, est destinée au dépôt et à la diffusion de documents scientifiques de niveau recherche, publiés ou non, émanant des établissements d'enseignement et de recherche français ou étrangers, des laboratoires publics ou privés. 


\title{
LE PARLEMENT EUROPÉEN : TENSIONS ENTRE EFFICACITÉ INSTITUTIONNELLE ET DÉMOCRATIE
}

Nathalie Brack, Olivier Costa

\author{
La Découverte | « Hérodote»
}

2017/1 Nº 164 | pages 199 à 212

ISSN 0338-487X

ISBN 9782707194480

Article disponible en ligne à l'adresse :

http://www.cairn.info/revue-herodote-2017-1-page-199.htm

\section{Pour citer cet article :}

Nathalie Brack, Olivier Costa « Le Parlement européen : tensions entre efficacité institutionnelle et démocratie », Hérodote 2017/1 ( $N^{\circ} 164$ ), p. 199-212. DOI 10.3917/her.164.0199

Distribution électronique Cairn.info pour La Découverte.

(C) La Découverte. Tous droits réservés pour tous pays.

La reproduction ou représentation de cet article, notamment par photocopie, n'est autorisée que dans les limites des conditions générales d'utilisation du site ou, le cas échéant, des conditions générales de la licence souscrite par votre établissement. Toute autre reproduction ou représentation, en tout ou partie, sous quelque forme et de quelque manière que ce soit, est interdite sauf accord préalable et écrit de l'éditeur, en dehors des cas prévus par la législation en vigueur en France. Il est précisé que son stockage dans une base de données est également interdit. 


\title{
Le Parlement européen : tensions entre efficacité institutionnelle et démocratie
}

\author{
Nathalie Brack ${ }^{1}$ et Olivier Costa ${ }^{2}$
}

La montée en puissance du Parlement européen (PE) est un phénomène abondamment étudié, notamment en lien avec le déficit démocratique de l'Union européenne [Corbett, 1998; Costa, 2001; Rittberger 2005]. Elle a été abordée tant du point de vue des facteurs explicatifs que de celui des conséquences sur le fonctionnement global du système politique européen [Reh, 2012]. Cette littérature a mis en évidence la capacité d'adaptation de l'institution à son environnement institutionnel, et en particulier son habilité à modifier ses méthodes de travail afin de maximiser son influence. Elle a aussi largement évoqué la question de l'impact de cette recherche d' 'efficacité» sur le caractère démocratique du système politique européen et du fonctionnement même de l'assemblée, en considérant souvent que les deux notions sont antithétiques [Ripoll-Servent, 2013].

Le rôle joué par l'organisation du travail parlementaire dans cette évolution reste un angle mort de la littérature. Alors que le règlement intérieur structure $d e$ facto le fonctionnement quotidien de l'institution et détermine les règles du jeu pour ses acteurs, aucune étude systématique n'en a été faite. L'objet de cet article est donc de comprendre comment et pourquoi les députés réforment ces règles et d'analyser les conséquences de ces réformes, notamment pour ce qui a trait à la démocratie dans l'institution.

Sur la base d'une analyse de l'évolution du règlement intérieur du PE entre 1979 et 2016, nous voulons démontrer que les députés cherchent, à travers ces

1. Chercheuse FNRS, Cevipol et IEE, Université libre de Bruxelles.

2. Directeur de recherche CNRS, Centre Émile Durkheim, Sciences Po-Bordeaux et directeur d'études, Collège d'Europe, Bruges. 
réformes constantes, à accroître l' «efficacité législative» de l'assemblée, afin de renforcer sa place dans le système politique de l'UE. L'argument de l'«efficacité» du travail parlementaire est progressivement devenu un objectif en soi et a pris le pas sur celui de liberté des parlementaires. Ces modifications ont conduit à une pacification de la délibération, grâce à la montée en puissance des organes hiérarchiques et des groupes politiques, à la réduction des espaces de libre débat et à un encadrement de plus en plus étroit du comportement et des droits des élus individuels.

Il ne s'agit pas ici d'adopter une approche normative des concepts d'efficacité, de performance, d'influence ou d'intérêt institutionnel, mais, au contraire, de souligner les enjeux qui entourent leur définition au sein de l'assemblée, à l'occasion des débats sur la réforme du règlement intérieur. Nous voulons montrer que, depuis le milieu des années 1980, les hiérarques et les responsables des grands groupes ont imposé leur lecture de l'intérêt institutionnel et des évolutions souhaitables du travail parlementaire, en prônant la restriction des libertés individuelles des députés au nom de ce qu'ils considèrent être l'intérêt de l'institution.

Depuis 1979, année de la première élection du PE au suffrage universel direct, le règlement intérieur de l'assemblée a été modifié à de très nombreuses reprises. Sans compter les changements mineurs intervenus au fil du temps, on ne recense pas moins de dix-sept éditions du règlement en trente ans (1979-2009). Consécutivement à l'entrée en vigueur du traité de Lisbonne, le règlement a une nouvelle fois été révisé en profondeur le 15 juin 2010 et est l'objet de nouvelles modifications sous la législature actuelle ${ }^{3}$. Ce document a gagné en complexité et est devenu de plus en plus précis afin d'adapter le fonctionnement du PE à ses nouvelles compétences. Alors qu'en avril 1979 le règlement comptait 42 pages, 54 dispositions et 2 annexes, à l'heure actuelle (juillet 2016), il contient 230 articles et 21 annexes, et compte, au total, 300 pages.

Vu l'ampleur du document, retracer et analyser de façon systématique l'ensemble de ces changements est impossible dans le cadre limité de cet article. Nous nous concentrerons ici sur l'évolution des dispositions ayant trait à quatre questions centrales pour la problématique qui nous intéresse: les droits individuels des députés, la régulation du comportement des élus, le poids des organes dirigeants et des groupes politiques et, enfin, l'organisation des débats.

3. Les nouvelles règles encadrant le comportement des députés dans leurs relations aux groupes d'intérêts ne sont pas prises en compte dans cet article. 


\section{Un encadrement plus rigoureux du comportement individuel des élus}

La pacification de la délibération résulte d'abord d'un encadrement de plus en plus rigoureux de la conduite des parlementaires en séance. L'analyse de l'évolution du règlement intérieur montre que les députés (ou, du moins, la majorité favorable à cette vision des choses) ont choisi de sacrifier progressivement leur liberté individuelle afin de privilégier l'efficacité du travail parlementaire et la capacité de l'assemblée à tirer profit de ses pouvoirs en respectant les délais et majorités imposés par les traités.

Les députés continuent certes à jouer un rôle clé dans le fonctionnement du $\mathrm{PE}$, en raison d'une logique de division du travail particulièrement poussée. Les textes, législatifs ou non, sont élaborés par un nombre limité d'acteurs: rapporteur, représentant le groupe qui a obtenu la responsabilité du rapport, et rapporteurs «fantômes» ou coordinateurs représentant les autres groupes. Ils disposent d'une réelle influence sur le résultat final. La montée en puissance des «trilogues» entre la Commission, le Conseil et le PE n'a pas remis en question ce rôle, puisque c'est, là encore, une poignée de parlementaires qui mène la négociation au nom de l'assemblée. Il est donc possible pour des députés, même backbenchers, d'avoir un rôle considérable dans le fonctionnement du PE, mais uniquement lorsqu'ils sont en situation de pouvoir, c'est-à-dire lorsqu'ils sont rapporteur, rapporteur «fantôme» ou coordinateur. Néanmoins, leur comportement en commission ou en plénière est contraint par des règles de plus en plus sévères. En outre, les élus qui ne remplissent pas les conditions pour accéder à ces positions de pouvoir (par exemple, les non-inscrits, les députés insuffisamment impliqués ou une partie des eurosceptiques) voient leur capacité d'action se réduire sans cesse.

En effet, les droits ouverts à un seul élu ont connu un déclin discret mais constant. Aujourd'hui, le règlement reconnaît à tout parlementaire cinq droits. Le premier est celui de poser une question écrite aux autres institutions de l'UE; il s'agit d'une ressource importante pour obtenir des informations, contrôler l'action de la Commission et du Conseil ou encore démontrer à ses électeurs ou à son parti que l'on est actif sur une thématique donnée. Certains membres, notamment eurosceptiques, font un usage extensif de cette procédure. En deuxième lieu, tout député peut également intervenir durant l'heure des questions. Le nombre de questions et de questions complémentaires a toutefois été progressivement réduit, pour tenir compte de l'inflation des effectifs (voir infra). De même, une réforme de juillet 2008 a encadré de façon plus stricte le contenu des questions : l'annexe III prévoit que les questions avec demande de réponse écrite doivent présenter un intérêt général, être concises (pas plus de 200 mots), contenir une demande compréhensible, ne pas avoir trait à des questions strictement personnelles ni contenir des propos insultants. Depuis septembre 2014, le nombre de questions 
a par ailleurs été limité et les pouvoirs du président ont été accrus, puisqu'il statue désormais sur la recevabilité des questions. Certains députés les utilisaient en effet comme plateforme d'expression ou évoquaient des problématiques très spécifiques à leur circonscription, sans rapport avec les activités de l'Union. Un élu a, en troisième lieu, le droit de faire une explication de vote, par voie écrite ou orale (voir infra). Un député peut aussi intervenir pour fait personnel, c'est-à-dire rectifier ses propres déclarations ou réfuter des propos tenus à son égard au cours des débats. Enfin, un élu peut proposer une motion de résolution sur un sujet entrant dans les compétences de l'UE. Bien que la procédure aboutisse rarement, elle permet à des députés d'accroître leur visibilité, notamment vis-à-vis de leurs électeurs.

Toutes ces possibilités offrent aux élus une certaine marge de liberté en séance plénière, mais la dynamique est à leur réduction. Jusqu'à récemment, un élu pouvait aussi prendre l'initiative d'une déclaration écrite qui, si elle recueillait les signatures de la majorité des membres du PE, était transmise aux institutions mentionnées. Les chances de succès étaient faibles, mais la procédure permettait aux députés de jouir d'une certaine visibilité. Cette possibilité n'est désormais ouverte qu'à dix députés, issus de trois groupes politiques au moins. En outre, au début des années 1990, les députés jouissaient de deux droits dont la portée était autrement plus importante: le dépôt d'amendements en séance plénière et la demande de renvoi d'un texte en commission. Aujourd'hui, les amendements ne peuvent être présentés en plénière que par la commission compétente au fond, un groupe politique ou quarante députés au moins; un député ne peut plus déposer d'amendement que dans la commission dont il est membre. De même, la demande de renvoi en commission qui permet, si elle est approuvée, de suspendre le débat et le vote sur un texte est désormais l'apanage des groupes politiques ou de quarante députés au moins.

L'impact et la portée des techniques d'obstruction ont été aussi progressivement réduits. Dans de nombreux parlements, les membres peuvent développer des stratégies dilatoires visant à prolonger indéfiniment les débats, à déposer un grand nombre d'amendements ou à abuser des motions de procédure. Ces outils ne sont plus efficaces au PE: la multiplication d'interventions en plénière n'est plus possible et le dépôt massif d'amendements ne permet pas de repousser significativement l'adoption d'un rapport, compte tenu des pouvoirs qui reviennent au Président pour décider ou non de les mettre aux voix. L'usage des motions de procédure a été encadré et est souvent restreint aux groupes ou à quarante élus, par exemple pour la vérification de quorum, l'inadmissibilité ou la clôture des débats. 


\section{Une régulation stricte du comportement des députés}

La présence dans les rangs de l'assemblée d'un nombre croissant de députés désirant faire entendre leur opposition à l'intégration européenne ou se servir du PE comme d'une tribune a également suscité une réaction des tenants de la rationalisation du fonctionnement parlementaire. Depuis la fin des années 1980, le contrôle des députés est ainsi devenu un enjeu récurrent des réformes du règlement. De manière presque systématique, la survenance de troubles en plénière a entraîné un durcissement des dispositions du règlement relatives au comportement des élus. La première réforme a eu lieu après deux incidents de ce type. Le premier, en octobre 1988, était le fait du révérend Ian Paisley, leader du Parti unioniste démocrate nord-irlandais, qui créa un scandale lors de la visite du pape Jean-Paul II au PE. L'année suivante, des élus du Groupe technique des droites européennes (extrême droite) protestèrent bruyamment contre leur exclusion des organes hiérarchiques des délégations interparlementaires, entrainant une rixe et une suspension de séance. En conséquence de ces événements, les députés ont attribué au président le pouvoir de rappeler à l'ordre le fauteur de troubles par deux fois avant de prononcer son expulsion immédiate de la salle et de proposer un vote de censure à son encontre; en cas d'approbation par l'assemblée plénière, le député fautif était exclu pour une période allant de deux à cinq jours. Malgré cette réforme, d'autres incidents eurent lieu. En juin 1995, de nombreux eurodéputés réagirent avec virulence à l'annonce par le président français Jacques Chirac de la reprise des essais nucléaires dans le Pacifique, alors même que celui-ci devait s'adresser au PE dans le cadre de la présidence française du Conseil. Son discours devant l'assemblée fut interrompu par des cris, sifflements et invectives, et une part significative des membres refusa d'applaudir son discours ${ }^{4}$. Compte tenu de l'ampleur du mouvement de protestation, qui avait mobilisé toute la gauche, il n'y eut pas de sanction individuelle et les initiatives visant à réviser une nouvelle fois le règlement intérieur n'aboutirent pas.

Une deuxième réforme importante eut lieu en conséquence de plusieurs incidents causés par des élus hostiles à l'intégration européenne, notamment après l'élargissement de 2004. L'assemblée a ainsi connu une session particulièrement troublée en janvier 2005, lorsque la campagne dite «Not in my name» (pas en mon nom) menée par des députés eurosceptiques polonais et britanniques perturba le lancement de la campagne d'information officielle sur la Constitution européenne,

4. Jean Quatremer titra ainsi dans Libération: «Cris, protestations, calicots et même quelques horions, c'est une véritable bronca parlementaire qui a accueilli Jacques Chirac venu dresser le bilan de la présidence française », 12 juillet 1995. 
entraînant agitation et échange de coups de poing dans l'hémicycle et en marge de celui-ci.

Les instances hiérarchiques du PE prirent conscience à l'occasion de ces événements qu'elles étaient désarmées face aux élus violents. Les sanctions existantes (exclusion de la plénière et, de fait, privation du droit de vote) étaient rarement appliquées et difficilement applicables, dans la mesure où elles portaient atteinte à l'exercice même du mandat représentatif des députés. Les dispositions encadrant la conduite des membres furent ainsi profondément réformées, du point de vue des principes qu'ils doivent respecter, des sanctions dont ils peuvent faire l'objet et de l'autorité compétente pour les prononcer. Le règlement révisé dispose que «le comportement des députés est inspiré par le respect mutuel, repose sur les valeurs et principes définis dans les textes fondamentaux de l'Union européenne, préserve la dignité du Parlement et ne doit pas compromettre le bon déroulement des travaux parlementaires ni la tranquillité dans l'ensemble des bâtiments du Parlement ». Une annexe (XV) a été ajoutée au règlement afin de préciser l'interprétation de cette disposition; elle prévoit une extension de la responsabilité des députés à l'ensemble de leur personnel et à tous les locaux du PE. De nouvelles sanctions ont, en outre, été introduites. Le Président peut désormais, en cas de trouble exceptionnellement grave ou de violation des principes de comportement, sanctionner le député sans soumettre cette décision au vote. La palette des sanctions est large: blâme; perte du droit à l'indemnité de séjour pour une durée pouvant aller de deux à dix jours; suspension temporaire, pour une durée de deux à dix jours consécutifs, de la participation à l'ensemble ou partie des activités du PE, sans préjudice de l'exercice du droit de vote en séance plénière; soumission à la Conférence des présidents d'une proposition de suspension ou de retrait d'un ou de plusieurs mandats électifs occupés au sein du PE (article 166, 2014). Un projet de rapport (soumis au vote en plénière le 12 décembre 2016 - rapport Corbett) prévoit d'augmenter ces sanctions, passant d'un maximum de 10 à 30 jours de perte d'indemnité et de suspension temporaire, et inclut la possibilité d'interdire à l'élu sanctionné de représenter le PE dans tout forum interinstitutionnel pour une période pouvant aller jusqu'à un an.

Malgré ces sanctions, les responsables du PE se trouvent dans l'incapacité pratique de mettre un terme aux comportements outranciers d'une partie des députés, extrémistes de gauche ou de droite, autonomistes ou eurosceptiques. Ces parlementaires donnent la priorité à la défense, par toutes les voies possibles, de leurs positions et de leurs idées, et n'estiment pas devoir se conformer à ce que les autorités du PE attendent d'eux. Les sanctions n'ont donc qu'une portée limitée à leur égard. Si elles consistent en des blâmes ou pénalités financières, elles apportent aux élus la publicité qu'ils recherchent (comme cela a été le cas pour le leader du UKIP Nigel Farage). Si elles vont au-delà, et prennent la forme d'une interdiction 
de participation aux activités de l'assemblée, elles font de ces parlementaires des martyrs et posent le problème de la légitimité de la mise à l'écart de députés régulièrement élus. C'est d'ailleurs en référence aux contraintes excessives qui pèsent sur leur comportement que ces élus justifient habituellement leurs dérapages [Brack, 2014].

Les débats sur l'organisation du travail parlementaire voient ainsi s'opposer deux conceptions du rôle de l'assemblée, parlement de travail vs parlement de parole. Elles renvoient à deux approches de l'impératif démocratique dans l'Union: la démocratie par le Parlement et la démocratie au sein du Parlement. La première met l'accent sur la démocratisation du fonctionnement de l'Union par la maximisation de l'influence du PE, et donc la rationalisation de ses activités et la recherche de l'efficacité. La seconde insiste sur le caractère démocratique du fonctionnement du $\mathrm{PE}$, et promeut un parlement de la libre parole, fondé sur des principes de pluralité et de représentativité et favorisant l'expression de toutes les sensibilités.

\section{La montée en puissance des organes hiérarchiques et des groupes politiques}

Les réformes du règlement ont aussi contribué à conférer un rôle central aux organes dirigeants du PE - notamment la Présidence et la Conférence des présidents - dans le fonctionnement de l'assemblée. Ils prennent en effet toutes les décisions importantes relatives à l'organisation du travail parlementaire et assurent l'essentiel de la «rationalisation» de son fonctionnement [Corbett et al., 2016; Costa et Brack, 2014].

Le Président a vu son rôle évoluer dans le sens d'un élargissement constant de ses pouvoirs [Costa, 2013, 2015]. En plus de sa fonction de représentation et de ses prérogatives dans la mise en œuvre des pouvoirs législatifs et budgétaires, il joue un rôle de direction du PE en présidant les séances plénières, la Conférence des présidents et le Bureau. Il assure aussi le respect et l'application du règlement intérieur à travers la direction de l'ensemble des activités de l'assemblée et de ses organes. La portée de sa fonction de direction a été élargie au cours de la $6^{\mathrm{e}}$ législature (2004-2009) par une interprétation du règlement destinée à lutter contre les manœuvres dilatoires de certains élus eurosceptiques. Le règlement stipule dorénavant qu'il dispose de tous les pouvoirs pour présider aux délibérations du PE et pour en assurer le bon déroulement: il peut mettre fin aux recours excessifs aux manœuvres dilatoires telles que rappels au règlement, motions de procédure, ou explications de vote, et il décide des sanctions à imposer aux élus en cas de non-respect des règles de conduite. La probabilité accrue pour un élu de se faire priver de la parole ou sanctionner a permis de limiter nettement les comportements d'obstruction dont une minorité s'était fait une spécialité. 
La Conférence des présidents est l'instance hiérarchique la plus importante du PE et celle qui a connu les plus fortes évolutions. Avant l'entrée en vigueur du traité de Maastricht (1993), il existait un «Bureau élargi», composé du Bureau (Président, vice-présidents et questeurs), des présidents des groupes politiques et de deux élus non inscrits (dépourvus de droit de vote). La réforme du règlement de 1993 a institué la Conférence des présidents, organe plus restreint, comprenant uniquement le Président, les présidents des groupes politiques et, depuis 2007, un élu non inscrit. Elle a de larges pouvoirs, puisqu'elle statue sur l'organisation des travaux et la programmation législative, autorise les rapports d'initiative et organise la concertation avec la société civile. La réforme de 1993 a également revu son mode de décision en introduisant le principe de pondération des voix des présidents de groupe en fonction des effectifs de leur formation respective; si un consensus ne peut être atteint, la Conférence procède à un vote pondéré.

Si toutes ces modifications formelles sont $a$ priori mineures, le rôle des organes hiérarchiques s'est fortement intensifié, pour faire face à l'hétérogénéité du PE et assurer une coordination politique de ses activités au plus haut niveau. Ces évolutions ont également contribué à renforcer l'influence des deux principaux groupes sur le pilotage des activités du PE. Sauf mésentente passagère (comme durant la législature 1999-2004), les groupes PPE (Parti populaire européen) et S \& D (Socialistes et démocrates) se partagent la présidence (en organisant une alternance concertée à mi-mandat), détiennent la majorité des postes de viceprésidents (au titre de la règle D'Hondt) et peuvent imposer leur point de vue au sein de la Conférence des présidents, du fait de la pondération des voix qui leur assure une large majorité. Depuis les élections européennes 2014 et la désignation de Jean-Claude Juncker en tant que président de la Commission avec le soutien des groupes PPE, S \& D et ALDE (Alliance des libéraux et des démocrates pour l'Europe), la coopération entre ces formations s'est renforcée. Elles composent désormais au PE un «bloc», qui apporte un soutien de principe aux initiatives importantes de la Commission. Cette nouvelle configuration politique se traduit par une coopération accrue s'agissant du fonctionnement de l'assemblée, dans un contexte d'inflation des effectifs eurosceptiques.

Les groupes politiques ont également vu leur rôle s'accroître, au détriment des droits des élus non inscrits et des députés individuels. Depuis 1979, les références du règlement au rôle des groupes se sont multipliées; parallèlement, le nombre d'élus à réunir pour exercer certains droits ouverts aux groupes a connu une inflation continue. En 2016, les candidats à la présidence du PE peuvent être proposés par un groupe (quelle que soit sa taille) ou quarante députés (sur 751, soit $5,33 \%$ ); cette proportion était de 5,05\% en 2004, de 4,59\% en 1994 et seulement de $2,44 \%$ en 1981 . De même, le droit de poser une question orale avec débat est attribué à une commission, un groupe politique ou, là encore, quarante élus (soit 
$5,33 \%$ ); en 1991, ce même droit était ouvert à 1,35\% des élus. Aujourd'hui, les groupes politiques exercent une forme de monopole sur les activités législatives et non législatives du PE et contrôlent l'accès aux ressources telles que la mise à l'agenda, l'allocation des rapports et des responsabilités.

Les réformes ont en particulier touché les députés non inscrits, pour la plupart eurosceptiques. Depuis les années 1980, ils ont vu leurs droits se réduire comme peau de chagrin, à mesure que les groupes politiques, en particulier les deux plus grands, renforçaient leur rôle dans le fonctionnement du Parlement et que les conditions pour former un groupe se durcissaient ${ }^{5}$. En raison de leur position sur la scène politique nationale et de leurs orientations idéologiques, les non-inscrits ne peuvent ou ne veulent pas rejoindre un groupe existant et ne parviennent pas à satisfaire aux conditions pour en former un autre. Dans la plupart des cas, les non-inscrits n'arrivent pas à agir de façon coordonnée, en raison de l'élévation constante du seuil à atteindre pour prendre une initiative formelle (généralement quarante élus). Comme tous les élus, ils ont souffert de la réduction des droits reconnus par le règlement aux députés individuels; en outre, le temps de parole qui leur était dédié a été fortement réduit lors de la réforme du règlement consécutive à l'adoption du traité de Maastricht. Auparavant, une première fraction du temps de parole était divisée équitablement entre tous les groupes et une seconde était répartie entre ces derniers en fonction de leur taille; les non-inscrits, considérés dans ce calcul comme un groupe, bénéficiaient d'un temps de parole double afin de tenir compte de la diversité de leurs orientations politiques. Depuis 1993, cette prime a été abolie.

Plus récemment, les droits des élus non inscrits ont à nouveau été affaiblis. Les deux plus grands groupes ont, tout d'abord, réclamé une réduction de leur représentation (sans droit de vote) à la Conférence des présidents; depuis 2007 ils n'envoient plus qu'un élu, contre deux jusqu'alors. La réforme du règlement du 15 juin 2010 a encore réduit la portée de ce droit: désormais, ce ne sont plus les non-inscrits qui choisissent leur délégué, mais le président du PE, qui peut ainsi choisir un député conciliant. Les non-inscrits sont également écartés, depuis 2009 , des réunions très stratégiques des «coordinateurs». Composées des élus qui chapeautent les délégations de chaque groupe au sein d'une commission parlementaire donnée, elles décident de l'allocation des rapports, des priorités politiques et de l'agenda des commissions.

Globalement, on a assisté à une marginalisation des non-inscrits, provoquant un sentiment d'impuissance politique chez ces élus. La question de leur discrimination

5. À partir de 1999, il est devenu impossible de former un groupe ne comprenant des députés que d'un seul État membre. Depuis 2009, le seuil est d'au moins vingt-cinq députés, en provenance d'un quart des États membres. 
est récurrente au PE. Les non-inscrits estiment qu'ils devraient bénéficier des mêmes droits que les autres élus, au nom de l'égalité, du pluralisme et des droits de l'opposition politique, mais les leaders du PE considèrent que l'efficacité du travail parlementaire est la meilleure garantie de la démocratie. Cette vision l'emporte souvent: chaque renforcement des droits des non-inscrits s'est accompagné d'autres réformes du règlement visant à réduire leur marge de manœuvre [Settembri, 2004].

\section{La réduction progressive des espaces de libre débat}

Les débats au sein du PE sont souvent considérés comme ternes et soporifiques en raison des multiples contraintes qui s'y appliquent: multilinguisme et interprétation, technicité des textes, absence de clivage clair entre majorité et opposition, forte spécialisation des élus, rôle prédominant du travail en commission, importance des négociations entre les groupes en marge de l'hémicycle, déconnexion entre le moment du débat et celui du vote [Costa, 2001]. Toutefois, les modalités de la délibération sont aussi le résultat des choix opérés par les députés. Au nom d'une certaine conception de l'efficacité du travail parlementaire, ils ont choisi d'allouer le temps de session de façon stratégique [Rasmussen et Toshkov, 2011] et de combattre toute forme d'obstruction, au détriment de la vivacité et de la spontanéité des débats.

Ainsi, depuis 1979, l'organisation des travaux en plénière a connu une évolution tendant vers une limitation de plus en plus stricte du temps de parole et une codification accrue du déroulement de la séance, qui suit un programme minuté. Les possibilités pour les élus de prendre la parole ou d'obtenir un temps de parole supplémentaire ont été progressivement limitées. En raison d'abus, les interventions sur le procès-verbal et l'ordre du jour, les motions de procédure et les interventions pour «fait personnel» ont été progressivement encadrées. Le temps de parole qui s'y attache est ainsi passé de trois à une minute par élu. En outre, pour les rappels au règlement, les députés sont désormais tenus de citer immédiatement la disposition qu'ils entendent invoquer.

L'heure des questions constitue un autre espace potentiel de libre débat. Cette procédure a été introduite consécutivement à l'adhésion du Royaume-Uni (1974), sur le modèle du Question time de la Chambre des communes. Elle permet à tout député de poser oralement une question aux représentants de la Commission et du Conseil. Jusqu'au début des années 1990, elle pouvait être suivie d'un débat d'une heure à la demande d'un groupe politique ou d'au moins sept députés (article 61,1991), mais cette possibilité a été supprimée après l'adoption du traité de Maastricht. Le temps de parole alloué pour poser une question a été réduit, de même que le nombre de questions. Enfin, la possibilité de question orale sans débat a été supprimée. 
Les «débats sur des problèmes d'actualité, urgents et d'importance majeure» constituaient une autre part importante de la délibération plénière dans les années 1980. Ils étaient un dérivatif pour des députés européens quasiment dépourvus de pouvoirs législatifs et leur permettaient d'affirmer le PE comme un acteur clé de l'espace public européen en voie de constitution. Dans les années 1990, les élus pouvaient délibérer dans ce cadre durant trois heures sur cinq sujets à chaque session plénière. Ces débats étaient beaucoup plus spontanés et passionnés que les débats législatifs, en raison des thématiques évoquées et des contraintes moindres en termes de prise de parole et de majorité à atteindre. Afin de donner plus d'espace aux travaux législatifs, les débats d'actualité ont été remplacés en 2002 par des «débats sur des cas de violation des droits de l'homme, de la démocratie et de l'état de droit», limités à trois sujets et une heure par session. Afin de compenser partiellement les effets de cette réforme, la possibilité a été introduite d'organiser, une fois par période de session, un débat extraordinaire, d'une durée d'une heure, sur un thème majeur concernant la politique de l'Union.

Les explications de vote fournissent une dernière possibilité aux élus d'intervenir en séance plénière. Là encore, le temps de parole prévu par le règlement est passé d'une minute et demie à une minute pour un député individuel, et de trois à deux minutes pour un groupe politique. Depuis 1994, le Président peut aussi placer ces explications après le vote final: les députés ne peuvent alors plus espérer rouvrir les débats et interviennent devant un hémicycle désert.

L'encadrement extrême des activités des élus en séance plénière suscite des polémiques comparables au statut des non-inscrits. Le clivage entre partisans et opposants à la régulation des comportements a été particulièrement sensible lors des débats sur la réforme du règlement occasionnée par l'entrée en vigueur du traité de Lisbonne ( $1{ }^{\text {er }}$ décembre 2009). Les représentants des grands groupes soulignaient la nécessité de procéder à de nouvelles réformes : «Le Parlement aura alors davantage de pouvoirs législatifs et nous devons nous préparer à placer l'activité législative au cœur de notre travail ${ }^{6}$.» Les membres de petits groupes dénonçaient cette vision des choses, considérant que les réformes proposées tendaient à «transformer notre parlement en une machine bureaucratique, où le rôle des députés, des groupes minoritaires et même des commissions doit être soumis au pouvoir décisionnel croissant, partiellement arbitraire, de la Conférence des présidents et de l'administration ${ }^{7} »$.

6. Extraits du débat du 5 mai 2009 sur la révision générale du règlement (rapport A6-0273/2009 R. Corbett); interventions de Jo Leinen (S \& D) et Monica Frassoni (Verts/ALE) respectivement.

7. Extraits du débat du 5 mai 2009 sur la révision générale du règlement (rapport A6-0273/ 2009 R. Corbett); interventions de Jo Leinen (S \& D) et Monica Frassoni (Verts/ALE) respectivement. 
Afin de remédier à l'atonie des débats tout en limitant les manœuvres dilatoires, la possibilité d'interventions d'une minute a été introduite dans le règlement en 2002. À l'instar de ce qui se pratique au Congrès américain, l'agenda prévoit une séquence de 30 minutes au début de chaque période de session permettant à tout élu d'intervenir une minute sur un sujet qu'il estime important. La réforme de 2009 a introduit par ailleurs la procédure du «carton bleu»: tout député peut le lever à l'intention du président de séance afin de poser une question pendant l'intervention d'un orateur, si ce dernier en est d'accord et si le Président estime que cela n'est pas de nature à perturber le débat.

\section{Conclusion}

L'analyse de l'évolution du règlement intérieur du PE montre qu'un train constant de réformes a pris place depuis le milieu des années 1980, au nom de l'«efficacité» du travail parlementaire. Les organes de direction ont vu leur pouvoir s'étendre sans cesse: le Président et la Conférence des présidents ont aujourd'hui un rôle beaucoup plus important qu'il y a quelques années et sont mieux à même d'assurer la coordination politique interne. Cette évolution a renforcé l'influence des deux grands groupes politiques, qui dominent les deux organes, tandis que le rôle individuel des députés a été réduit de manière significative. Enfin, les modalités de la délibération en séance plénière ont été de plus en plus encadrées, jusqu'à ne laisser qu'une faible marge de manœuvre aux députés.

Ces évolutions sont considérées par leurs tenants comme un moyen d'accroître la capacité du PE de profiter de ses pouvoirs formels, mais aussi de garantir sa «crédibilité » dans les négociations interinstitutionnelles. Le travail conjoint des membres des deux groupes majoritaires permet en effet au PE de respecter les conditions de majorité et de délais prévues par les traités pour les procédures législatives et budgétaires, et de définir une stratégie institutionnelle claire dans ses relations avec la Commission, le Conseil et le monde extérieur. C'est à travers la promotion de leur assemblée que les tenants de cette approche cherchent à accroître leur propre pouvoir individuel et leur visibilité.

En raison de ces évolutions, le $\mathrm{PE}$ est devenu une assemblée plus homogène et moins représentative, dans ses activités et ses décisions, de la diversité idéologique de ses membres. Il est également moins à même de rendre les enjeux de l'intégration européenne lisibles aux yeux des citoyens; les députés se sont concentrés sur des impératifs fonctionnels et tiennent encore peu compte de l'image publique de leur institution. La montée en puissance des eurosceptiques, qui représentent plus de $20 \%$ des effectifs aujourd'hui, s'est traduite par un renforcement de l'alliance entre les socialistes et les démocrates-chrétiens; il a accentué l'image unanimiste 
de l'assemblée et suscite l'incompréhension dans les États où la bipolarisation de la vie politique est la norme.

Bien qu'elle soit une constante de l'évolution du PE depuis les années 1980, la rationalisation de son fonctionnement suscite un double clivage parmi les députés. Le premier oppose les backbenchers aux hiérarques et aux députés les plus systématiquement investis dans le travail législatif, en raison de leurs appréciations divergentes des priorités stratégiques et des moyens d'améliorer le fonctionnement du PE. Dans une logique de vases communicants, les seconds ont largement bénéficié du processus de rationalisation en termes d'influence, au détriment des premiers. La généralisation des «trilogues» en matière législative et de l'adoption des textes dès la première lecture n'a fait qu'accentuer cette opposition, en distinguant clairement les députés qui sont désormais régulièrement impliqués dans les négociations avec la Commission et le Conseil et ceux qui n'y participent jamais [Costa, Dehousse et Trakalova, 2011]. Un second clivage oppose les membres des «grands » groupes (PPE et S \& D, et dans une moindre mesure ALDE) à ceux des autres formations. Depuis le milieu des années 1980, les premiers assurent une sorte de cogestion des aspects organisationnels de l'assemblée et contribuent largement à imposer l'idée de rationalisation. Au nom du principe de répartition proportionnelle des ressources, les autres groupes bénéficient eux aussi de moyens et attributs, mais ils n'en subissent pas moins la loi du PPE et du S \& D, qui s'entendent bien souvent en amont des discussions. Ce clivage recouvre, en large partie, une opposition entre députés pro- et anti-européens. Les premiers privilégient la participation du PE au fonctionnement de l'Union et à l'approfondissement de l'intégration européenne, et sont disposés pour cela à favoriser l'émergence de positions majoritaires au sein de l'assemblée et une approche non conflictuelle des relations interinstitutionnelles. Les seconds se focalisent sur la dénonciation des travers de l'intégration européenne; compte tenu de leur situation structurellement minoritaire, ils privilégient les stratégies d'obstruction et de protestation, récusent la recherche du consensus interinstitutionnel et s'opposent à l'idée de rationalisation du fonctionnement du PE. Depuis 2014, l'émergence du «bloc» et la progression des eurosceptiques n'ont fait que renforcer cette tension et cliver un peu plus le PE mais, en l'état actuel des choses, la priorité reste à la recherche de ladite «efficacité» institutionnelle.

\section{Bibliographie}

ABÉLÈs M. (1992), La Vie quotidienne au Parlement européen, Hachette, Paris. BRACK N. (2014), L'Euroscepticisme au sein du Parlement européen. Stratégies d'une opposition anti-système au cœur des institutions, Larcier, Luxembourg. 


\section{HÉRODOTE}

CORBETT R. (1998), The European Parliament's Role in Closer European Integration, Macmillan, Londres.

CorbetT R., JACOBS F. et SHACKLETON M. (2016), The European Parliament, $7^{\text {e }}$ éd, John Harsher Publisher, Londres.

Costa O. (2015), «Le président du Parlement européen: un leader méconnu », in Blumann C. et PICOD F. (dir.), Annuaire de droit européen 2013, Éditions Panthéon Assas, Paris, p. 33-51.

Costa O. (2013), «The President of the European parliament», Il Filangieri (revue de l'ARSAE), vol. 2012-2013, «Le trasformazioni del ruolo dei Presidenti delle Camere», Ed. Jovene, Naples, p. 143-160.

Costa O. (2001), Le Parlement européen, assemblée délibérante, Éditions de l'Université de Bruxelles, Bruxelles.

Costa O., Dehousse R. et Trakalova A. (2011), «La codécision et les "accords précoces". Progrès ou détournement de la procédure législative?», Notes de la Fondation Notre Europe, $\mathrm{n}^{\circ} 84$, mars.

Costa O. et BRACK N. (2014), Le Fonctionnement de l'Union européenne, Éditions de l'Université de Bruxelles, Bruxelles.

Priestley J. (2008), Six Battles that Shaped Europe's Parliament, John Harper, Londres.

RASMUSSEN A. et TOSHKOV D. (2011), «The inter-institutional division of power and time allocation in the EP», West European Politics, vol. 34, $\mathrm{n}^{\circ} 1$, p. 71-96.

REH C. (2012), «European integration as compromise: Recognition, concessions and the limits of cooperation », Government and Opposition, vol. 47, n³, p. 414-440.

RIPOLL-SERVENT A. (2013), «Holding the European parliament responsible: policy shift in the data retention directive from consultation to codecision », Journal of European Public Policy, vol. 20, n 7, p. 972-987.

RitTBerger B. (2005), Building Europe's Parliament. Democratic Representation Beyond the Nation State, Oxford University Press, Oxford.

SAMUELSON Paul A. (1954), The Pure Theory of Public Expenditure, vol. 36, $\mathrm{n}^{\circ} 4$.

SETTEMBRI P. (2004), «When is a group not a political group? The dissolution of the TDI group in the European parliament », The Journal of Legislative Studies, vol. $10, \mathrm{n}^{\circ} 1$, p. $150-174$. 